\title{
Overview of the National Aeronautics and Space Administration's Nondestructive Evaluation (NDE) Program
}

\author{
Edward R. Generazio \\ Nondestructive Evaluation Sciences Branch \\ Langley Research Center \\ National Aeronautics and Space Administration \\ Hampton, Virginia 23681
}

\begin{abstract}
Abstract. NASA's Office of Safety and Mission Assurance sponsors an Agency-wide NDE Program that supports Aeronautics and Space Transportation Technology, Human Exploration and Development of Space, Earth Science, and Space Science Enterprises. For each of these Enterprises, safety is the number one priority. Development of the next generation aero-space launch and transportation vehicles, satellites, and deep space probes have highlighted the enabling role that NDE plays in these advanced technology systems. Specific areas of advanced component development, component integrity, and structural heath management are critically supported by NDE technologies. The simultaneous goals of assuring safety, maintaining overall operational efficiency, and developing and utilizing revolutionary technologies to expand human activity and space-based commerce in the frontiers of air and space places increasing demands on the Agencies NDE infrastructure and resources. In this presentation, an overview of NASA's NDE Program will be presented, that includes a background and status of current Enterprise NDE issues, and the NDE investment areas being developed to meet Enterprise safety and mission assurance needs through the year 2009 and beyond.
\end{abstract}

\section{NONDESTRUCTIVE EVALUATION PROGRAM}

\section{NDE Program Management}

The NASA Office of Safety and Mission Assurance (OSMA) NDE Program closely coordinates NDE activities among all interested NASA Headquarters Offices and the NASA field installations. The OSMA serves as the NASA focal point for OSMA 
Agency-wide NDE policy coordination and cooperative activities with other Government agencies, industry, educational, and nonprofit organizations. The Agency-lead, NASA Langley Research Center (LaRC), develops and maintains a coordinated and comprehensive Agency-wide NDE program. The tasks within the NDE Program are the responsibility of representatives at the designated field installations.

\section{NASA NDE Working Group (NNWG)}

The Nondestructive Evaluation (NDE) Working Group was established to provide a forum for Agency-wide cooperation to improve the effectiveness of communication and coordination in the NASA NDE community, promote technical integration, improve customer interfaces, and optimize the use of resources. The Working Group is under the sponsorship of OSMA and provides advice and assistance to the Agency in the areas of nondestructive evaluation, inspection, and testing (NDE/NDI/NDT).

\section{NDE Technical Program for Aero-Space Technology Human Exploration and Development of Space, Earth Science, And Space Science Enterprises}

The NDE Technical Program plans describe the top level and details of the Agency-wide NDE program for the fiscal years 1999 through 2002 (FY99-FY02). A concentrated effort has been made to efficiently address short-term critical mission assurance issues using a dynamic inter-Center NDE team (Rapid Response Team). NDE activities for the Space Transportation System (STS) and infrastructure recieve top priority, while mission assurance NDE activities for satellites and deep space probes are initiated to meet program plans and launch schedules. NDE activities for aeronautics are focused on airframe and engine structural integrity and operational health monitoring.

Several NDE task were completed this fiscal year with demonstrations of a costeffective, easy-to-use, reliable reflectometer for determining the emissivity of the Shuttle insulation blankets; a cable integrity tester that will verify electrical continuity through Shuttle signal conditioners without de-mating connectors; an ultrasonic system for determining engineering properties of composites; and the identification of NDE methodologies for assuring the structural and electronic integrity of Microelectromechanical Systems (MEMS) and high density electronic devices for Mar Microprobe and satellite systems. Also completed were the probability of detection (POD) specimen set for fracture control analysis and verification of POD capability for organizations inspecting NASA fracture critical space hardware, and the draft document "NDE requirements and Guidelines for 
Fracture Control Programs". An effort to develop a NDE process for grading the quality of Cadmium Zinc Telluride (CZT) to enhance and assure gamma and Xray sensitivity for International Focusing Optics Collaboration microCrab (InFOCuS) and Constellation X programs has been accelerated. The NDE Rapid Response Team (RRT) was utilized for the Solid Rocket Booster (SRB) Nozzle Anomalous Erosion; Space Station Window interlaminar scratches; Mars Microprobe circuit backbone integrity and ceramic shell characterization; and the B757 research aircraft lap joint inspection. The RRT has also addressed the Space Shuttle Main Engine (SSME) main combustion chamber liner oxidation.

In FY99 and FY00, there will be demonstrations of NDE methodology for monitoring the quality of the Friction Stir Welding process for the Super Light Weight Tank and aeronautic applications, and of the large lift-off eddy current probe for the detection of hidden corrosion of the Orbiter skin. NDE technology developments will be continued to assure integrity of cryogenic composite components, and enhance the utilization of advanced thermography on the Thermal Protection System and composite tankage of the X-33, SRB and External Tank Composite nose caps and nose cones, Space Shuttle Main Engine Nozzle, Space Station composite racks, and X-38 hybrid composite aeroshell.

New FY99-FY00 NDE activities are addressing: launch pad valve health monitoring, and the astronaut space adaptation syndrome. The development of a durable, harsh environment compatible, NDE sensor for monitoring environmentally hazardous aircraft engine NOx emissions will be initiated. A smart, real-time, whole field, optically-based, spin pit bladed disk damage detection system for reducing aircraft engine fan blade and blisk development and inspections times is being pursued. Process control NDE standards for Area Array Packaging (AAP) interconnect integrity are being developed to increase mission assurance of New Millennium Mars Exploration, and Deep Space Network programs. Increased mission assurance is being obtained in the Mars Observer Corrective Action Test Program (MOCATP) and Space Station Interim Control Module (ICM) by verifying pyrovalve ram o-ring seals to reduce the risk of spacecraft loss from leakage of hot pyrotechnic blow-by.

\section{Programmatic Technical Synergy}

Examples of technical synergy across programmatic lines are observed through the development of several advanced NDE technologies sponsored under the LaRC's Airframe Structural Integrity Program (ASIP) and Airframe Airworthiness Assurance (AAA) Programs, and OSMA NDE Program. The rotating self-nulling probe developed under ASIP for the detection of cracks in multi-layer aircraft skins has been adapted to quantitatively measure the electrical conductivity of the SSME combustor liner. The advanced thermal diffusivity imaging system, also developed under ASIP, is directly applicable to inspection issues of the SSME nozzle and composite flight hardware of the Bantum and X-33 programs. The optical simulation of electron emission (OSEE) surface contamination monitor was 
developed under the OSMA NDE program for the characterization of residual contamination present (due to the new requirement for environmentally friendly cleaners) in the reusable SRB. OSEE is being used in the Airframe Systems Base Program's development of a NDE system for the quantitative determination of bond strength. Distributed fiber optic sensors developed under ASIP for detection of corrosion in aging aircraft have been extended to measure strain, temperature, and the presence of environmental elements. These new fiber optic sensor systems form the technical basis for the X-33's integrated vehicle health monitoring (IVHM) system and the proposed Space Shuttle hydrogen leak monitoring systems of the Space Shuttle upgrade program.

The current status of NDE tasks is available at the web-site http://www-nesb.larc.nasa.gov

\section{NONDESTRUCTIVE EVALUATION TECHNOLOGY GAPS}

Current events often highlight the importance of using a system-thinking approach to address current and future issues that require the application of advanced NDE technologies. For example, serious damage resulted from the collision accident of the space station Mir "Spektr" module. In a space environment, the extent of the damage is difficult to assess. On Earth this is less of a problem because of extensive tools and better access are available. Unfortunately, in space these tools are not available. The development of spacebase NDE technologies needs to be accelerated to match mission requirements. Other examples, such as, the anomalous erosion of the Shuttle's Solid Rocket Booster Nozzle, and complications in the manufacturing of the structurally complex X-33 (Reusable Launch Vehicle demonstration vehicle) tankage exposed the need for advances in NDE technologies to address complex hybrid composite structures. The NDE state-of-the-art is significantly advanced for basic metal and composite structures applications. However, there is a major technology gap growing between current NDE capability and future NDE requirements for complex metallic and composite hybrid structures. The development of NDE technologies from concept to commercialization can take two to five years, therefore, this gap needs to be addressed early to enable sufficient inspection methodologies for the future.

\section{EVOLUTION OF NONDESTRUCTIVE EVALUATION TECHNOLOGY}

Beginning with the birth of radiography in the early 1900's, NDE was driven by the development the medical ultrasonics, eddy current, fluorescent penetrant and magnetic particle techniques of the 1960's. X-ray computed tomography, holography, laser ultrasonics, and shearography gained acceptance in the 1970- 
1980's. NDE technologies emerging from the 1990's are thermal diffusivity, magnetoptics, microwave, contamination monitoring, intra-cranial pressure monitoring, and NDE computational simulations.

The application of discrete point NDE measurements are generally costly. This is particularly true for aging systems that are being used beyond their design life. The missions of the future need to be more cost-effective throughout the life cycle and to reach this goal a different approach will be required. The approach of the future will utilize brilliant integrated vehicle health management systems that working together with autonomous agents or companions systems that consist of derivatives of existing NDE technologies, as well as, newly developed NDE technologies.

In the year 2015, it is envisioned that an enabling presence of nondestructive evaluation and health management systems will play a critical role in the development and utilization of advanced materials and structural aero-space systems. Statistical manufacturing and assembly process variations, harsh dynamic working environment, and impact damage will still exist in 2015. Multi-level and broad nondestructive evaluation approaches will be operational to meet these challenges. A global structural heath management system will serve the need to dynamically evaluate structural integrity of large and remote structural systems. This evaluation will be done with multidisciplinary-based probes developed for interrogating large structural components. The data acquisition, structural analysis and effects of anomalous materials and structural variations will be automated and controlled by a multi-level intelligent control system. The outputs will be primarily: time dependent probability-of-failure for predicting remaining life; percent of residual strength integrity; and risk. A primary question to be addressed by the health management system is: "If the structure fails, then what will be the outcome?"

Today, NASA is already implementing fiber optic strain and temperature monitoring sensor systems on aerospace structures. Fiber optic sensors for hydrogen monitoring are already being tested on the Space Shuttle. It will not be long before these IVHM systems become smart tele-sensors, neural-networked into a re-configurable, decision making, distributed intelligence.

The above global structural health management system ensures the ultimate structural integrated security for aerospace systems. A secondary NDE system is in place to autonomously identify, evaluate, and repair a wide range of damage or defect conditions in aerospace materials and structures. This inspection system is also multidisciplinary, utilizing intelligent micro-sized NDE sensors, multiple miniature robots, and micro-sized repair systems. Autonomous, self-healing smart structures also provided raw materials to make repairs, and integral materials sensors deform, possibly "electropiezomagneto-mechanically", the structure to redistribute load while the repair is being made and, subsequently, certified. The copious, generally volumetric, inspection data is internally fused and analyzed by small, near optical speed, fail-safe micro-computational systems that operate both at the individual sensor level and as an integrated computational decision making network that coordinates activities with the global inspection system. 
In order to reach this vision, investments in areas of smart health monitoring, telerobotic inspection and repair, NDE simulations (for design, process control, and inspection protocol), multi-mode data fusion, fatigue and residual life sensors, and quantitative bond strength are being pursued today.

\section{SUMMARY}

An overview of NASA's NDE Program has been presented covering background and status of current Enterprise NDE issues, and the NDE investment areas being developed to meet Enterprise safety and mission assurance needs in the year 2009 and beyond. 